\title{
Highly Efficient Genome Engineering in Bacillus anthracis and Bacillus cereus Using the CRISPR/Cas9 System
}

Yanchun Wang ${ }^{\dagger}$, Dongshu Wang ${ }^{\star \dagger}$, Xiaojing Wang, Haoxia Tao, Erling Feng, Li Zhu,
Chao Pan, Bowen Wang, Chunjie Liu*, Xiankai Liu* and Hengliang Wang*

State Key Laboratory of Pathogens and Biosecurity, Beijing Institute of Biotechnology, Beijing, China

Genome editing is an effective tool for the functional examination of bacterial genes and for live attenuated vaccine construction. Here, we report a method to edit the genomic DNA of Bacillus anthracis and Bacillus cereus using the clustered regularly interspaced short palindromic repeats (CRISPR)/CRISPR-associated protein (Cas)9 system. Using two prophages in $B$. anthracis as targets, large-fragment deletion mutants were achieved with rates of 100 or $20 \%$. In B. cereus, we successfully introduced precise point mutations into plcR, with phenotypic assays showing that the resulting mutants lost hemolytic and phospholipase enzyme activities similar to $B$. anthracis, which is a natural plcR mutant. Our study indicates that CRISPR/Cas9 is a powerful genetic tool for genome editing in the Bacillus cereus group, and can efficiently modify target genes without the need for residual foreign DNA such as antibiotic selection markers. This system could be developed for use in the generation of marker-free live anthrax vaccines or for safer construction of microbiological candidate-based recombinant $B$. cereus.

Keywords: CRISPR/Cas9, Bacillus cereus sensu lato group, Bacillus anthracis, Bacillus cereus, large genomic deletion, genomic site-specific mutagenesis

\section{INTRODUCTION}

Targeted genome modification technology refers to the alteration of specific sites within the genome. Continuous research and development has so far established several theories and methods to achieve this goal (Joung and Sander, 2013; Gersbach et al., 2014; Maeder and Gersbach, 2016). Clustered regularly interspaced short palindromic repeats (CRISPR)/CRISPR-associated protein (Cas)9 technology is a recently developed targeted genome modification approach that has been successfully applied to a wide variety of eukaryotic and prokaryotic cells (Cong et al., 2013; Chandrasekaran et al., 2017). The technique uses a length of RNA to identify target sites, making it simpler to design and implement than other methods. It also offers the following unparalleled advantages: (1) a high modification efficiency that does not usually require screening markers, (2) the capacity to simultaneously modify multiple sites using different single guide (sg) RNAs, and (3) the ability to change the target sequence recognition site by altering only a short RNA sequence within the specific identification domain (Zhang et al., 2014; Chandrasekaran et al., 2017).

The Bacillus cereus sensu lato group consists of 18 closely related sporulating Gram-positive bacteria: B. anthracis, B. cereus, B. thuringiensis, B. albus, B. cereus, B. cytotoxicus, B. luti, B. mobilis, B. mycoides, B. nitratireducens, B. pacificus, B. paramycoides, B. paranthracis, B. pseudomycoides, B. toyonensis, B. tropicus, B. wiedmannii, and 
B. weihenstephanensis (Rossi et al., 2017; Acevedo et al., 2019). The main representatives of this group are B. anthracis, B. cereus, and $B$. thuringiensis. The chromosomes of these three bacteria show very high genetic similarity and their rRNA sequences are almost identical, differing only within the expected range of variation for a single species. However, the three species show highly divergent pathogenicity (Guinebretière et al., 2008; Liu et al., 2015; Okinaka and Keim, 2016). B. thuringiensis shows specificity for insect larvae, while $B$. anthracis and $B$. cereus are human pathogens. Although $B$. anthracis-induced anthrax is often fatal, $B$. cereus is a conditional pathogen that causes various diseases in humans such as periodontitis, food poisoning, and acute ophthalmitis (Kotiranta et al., 2000; Argôlo-Filho and Loguercio, 2014; Granum, 2017; Pilo and Frey, 2018).

The modification of target genes is important for research into the pathogenic mechanisms of bacterial strains. In recent years, many successful methods have been developed, however, most rely on the principle of homologous recombination (Dong and Zhang, 2014; Wang et al., 2016), and B. anthracis, B. cereus, and $B$. thuringiensis have very low homologous recombination efficiencies (Plaut and Stibitz, 2015). Additionally, research to date has mainly focused on the deletion of target genes, so precise point mutations and the removal of large fragments have not been achieved. Therefore, it is necessary to develop a new method to improve the efficiency of genetic modification in these bacteria. CRISPR/Cas9 technology has been widely used in B. subtilis, and a variety of relatively mature technical protocols have been constructed (Altenbuchner, 2016; Westbrook et al., 2016; Li et al., 2018). However, comparable research in the Bacillus cereus group is relatively limited. To date, the CRISPR/Cas9 system has only been used to construct $B$. thuringiensis mutants, and no related work has been reported in B. anthracis or B. cereus (Guo et al., 2019; Tan et al., 2019).

In the present study, we developed a highly efficient genome engineering protocol for $B$. anthracis and $B$. cereus based on the CRISPR/Cas9 system. Selecting two prophages contained within the genome of $B$. anthracis as targets, we successfully obtained large-fragment deletion mutants, resulting in a successful modification rate close to $20 \%$ for $50 \mathrm{~kb}$ fragment deletions. When the method was applied to delete a smaller genomic fragment from B. anthracis, a $100 \%$ modification rate was achieved. In $B$. cereus, we successfully introduced a precise point mutation into $p l c R$, an important regulatory gene, and phenotype assays showed that mutants lost hemolytic and phospholipase enzyme activities. The protocol developed in this study will be a valuable tool for the generation of marker-free live anthrax vaccines and for the construction of safer microbiological candidate-based recombinant $B$. cereus.

\section{MATERIALS AND METHODS}

\section{Bacterial Strains and Growth Conditions}

All bacterial strains used in this study and their relevant characteristics are listed in Table 1. Bacteria were grown aerobically at 30,37 , or $42^{\circ} \mathrm{C}$. Escherichia coli strains were grown in Luria-Bertani (LB) broth and used as hosts for plasmid cloning.
TABLE 1 | Plasmids and strains used in this study.

\begin{tabular}{|c|c|c|}
\hline $\begin{array}{l}\text { Plasmids and } \\
\text { strains }\end{array}$ & Relevant characteristics & Source \\
\hline \multicolumn{3}{|l|}{ Plasmids } \\
\hline pJOE8999 & CRISPR-Cas9 vector; KanR & Altenbuchner, 2016 \\
\hline pJOE-Lam01 & $\begin{array}{l}\text { pJOE8999 with sgRNA-lam01 and } \\
\text { homologous arms of lam01 from B. } \\
\text { anthracis A16R, KanR }\end{array}$ & This study \\
\hline pJOE-Lam03 & $\begin{array}{l}\text { pJOE8999 with sgRNA-lam03 and } \\
\text { homologous arms of lam03 from B. } \\
\text { anthracis A16R, KanR }\end{array}$ & This study \\
\hline pJHRT & $\begin{array}{l}\text { pJOE8999 with sgRNA-plcR and } \\
\text { homologous arms of plcR from B. } \\
\text { cereus HN001, KanR }\end{array}$ & This study \\
\hline \multicolumn{3}{|c|}{ B. cereus group strains } \\
\hline B. anthracis A16R & $\begin{array}{l}\mathrm{pXO1} 1^{+} \mathrm{pXO} 2^{-}, \text {China vaccine } \\
\text { strain }\end{array}$ & This laboratory \\
\hline $\begin{array}{l}\text { B. anthracis A16R } \Delta \\
\text { lam01 }\end{array}$ & $\begin{array}{l}\text { A16R excision prophage } \\
\text { lambdaBa01 }\end{array}$ & This study \\
\hline $\begin{array}{l}\text { B. anthracis A16R } \Delta \\
\text { lam03 }\end{array}$ & $\begin{array}{l}\text { A16R excision prophage } \\
\text { lambdaBa03 }\end{array}$ & This study \\
\hline B. anthracis A16PI2 & $\begin{array}{l}\mathrm{pXO1} 1^{+} \mathrm{pXO} 2^{-}, \text {deriving from } \mathrm{A} 16 \\
\left(\mathrm{pXO} 1^{+} \mathrm{pXO} 2^{+}\right)\end{array}$ & Wang et al., 2011 \\
\hline B. cereus HN001 & Wild-type B. cereus & This laboratory \\
\hline B. cereus HN1M & $\begin{array}{l}\text { B. cereus HN001 with G640T } \\
\text { mutation in plcR }\end{array}$ & This study \\
\hline \multicolumn{3}{|l|}{ E. coli strains } \\
\hline $\mathrm{DH} 5 \alpha$ & Cloning strain & CWBIO, China \\
\hline SCS110 & $\begin{array}{l}d a m-/ d c m \text { - strain used to produce } \\
\text { unmethylated plasmid }\end{array}$ & Transgen, China \\
\hline
\end{tabular}

LB agar was also used for the selection of transformants. For competent cell preparation, B. anthracis strains were grown in brain heart infusion broth with the addition of $0.5 \%$ glycerol (BHIG; Becton, Dickinson and Company, Franklin Lakes, NJ, United States). Kanamycin was added to the growth media at a concentration of $50 \mu \mathrm{g} / \mathrm{ml}$ for $E$. coli and $25 \mu \mathrm{g} / \mathrm{ml}$ for both $B$. anthracis and $B$. cereus. To induce the expression of Cas9 protein, LB medium was supplemented with $25 \mu \mathrm{g} / \mathrm{ml}$ kanamycin and $0.4 \%$ mannose. Sheep blood agar plates and lecithin agar plates (LB agar supplemented with $1 \%(\mathrm{v} / \mathrm{v})$ egg yolk) were used to detect hemolysis and phospholipase activity, respectively.

\section{DNA Manipulation}

The preparation of plasmid DNA from E. coli, transformation of $E$. coli, and recombinant DNA techniques were carried out using standard procedures. E. coli $\mathrm{DH} 5 \alpha$ competent cells were obtained from CWBio (Beijing, China), while E. coli SCS110 competent cells were purchased from Transgene (Beijing, China). Recombinant plasmid construction was carried out in E. coli DH5a. B. anthracis A16R chromosomal DNA was isolated using a Wizard Genomic Purification Kit (Promega, Madison, WI, United States) according to the protocol for the isolation of genomic DNA from Gram-positive bacteria. B. anthracis A16R and $B$. cereus HN001 were electroporated with unmethylated plasmid DNA isolated from E. coli SCS110, and electrocompetent cells were prepared as previously described (Wang et al., 2018). 
TABLE 2 | Primers used in this study.

\begin{tabular}{|c|c|c|}
\hline Name & Sequence $\left(5^{\prime} \rightarrow 3^{\prime}\right)$ & Purpose \\
\hline Ulam03F & ACGCGTCGACTTACGGCAATGTTCCAAAG & PCR of homology arms for lambdaBa03 excision \\
\hline Ulam03R & ПTIGGTCTCATAATITACTGACCGTATTGCTAAG & \\
\hline Dlam03F & TाGGTCTCAATTATCGTTGATGTTATAAAAAG & \\
\hline Dlam03R & GCTCTAGACTCCAAACAAAGGTAAACTAGG & \\
\hline sg-lam03F & tacgAACTAAGAAGGATATTCCAA & Target sequence for lambdaBa03 excision \\
\hline sg-lam03R & aaacTTGGAATATCCTTCTTAGTT & \\
\hline lam03p1 & CCTGGGATTGATGATACGATGGC & PCR of lambdaBa03 excision mutant identification \\
\hline lam03p2 & TTGGTITCGACGTAACTGACCAAG & \\
\hline lam03p3 & CCAAAATCAGCTGTAGCGATATTC & \\
\hline lam03p4 & TATCCATATAATGAGTाIITCTGCПाT & \\
\hline lam03p5 & CCTTCCTCGGCTTCTTCCATTG & \\
\hline Ulam01F & ACGCGTCGACTAATTGCAAATAACGG & PCR of homology arms for lambdaBa01 excision \\
\hline Ulam01R & ПTIGGTCTCAAGAGGGGATATATTCCGCACAC & \\
\hline Dlam01F & ПTGGTCTCCCTCTTAGTAAAGAGAC & \\
\hline Dlam01R & GCTCTAGAGGATTCTCAGATITCAATC & \\
\hline sg-lam01F & tacgTTAGACCCTCTACTACCAAG & Target sequence for lambdaBa01 excision \\
\hline sg-lam01R & aaacCTTGGTAGTAGAGGGTCTAA & \\
\hline lam01p1 & TAAGCAATAATACATAGCAACAAACC & PCR of lambdaBa01 excision mutant identification \\
\hline lam01p2 & GTAATITCCCTTGGACAGCTG & \\
\hline lam01p3 & AAAGTGCAGCACCTACACTGAAAC & \\
\hline lam01p4 & AGTITTCGATGAACTCAATGGCATG & \\
\hline lam01p5 & ATATITCAAAGAAATAAAAGCCC & \\
\hline sg-plcRF & tacgAGGTGAATGCCTAGGGAAGT & Target sequence for site-specific mutagenesis of plcR \\
\hline sg-plcRR & aaacACTTCCCTAGGCATTCACCT & \\
\hline pJOEF & TAGTGTAGCCGTAGTTAGG & PCR identification of the introduction of pJHRT into HN001 \\
\hline pJOER & AAAGGGAATGAGAATAGTG & \\
\hline plcRHMF & CTTCTGTTGATAAAGGGCAAAGAAG & PCR of plcR mutagenesis identification \\
\hline plcRHMR & TTAAAGTGATTGCAGAAGGTGTA & \\
\hline
\end{tabular}

\section{Plasmids Construction}

All bacterial strains and plasmids used in this work are shown in Table 1, and PCR primers are listed in Table 2. Oligonucleotides for gRNA construction were designed using the online sgRNA design tool ${ }^{1}$. High-scoring 20-nucleotide (nt) sequences were selected.

To explore the feasibility of large chromosomal deletions using the CRISPR/Cas9 system in B. anthracis, we selected the shortest (lambdaBa03, $\sim 16.8 \mathrm{~kb}$ ) and longest (lambdaBa01, $\sim 50.5 \mathrm{~kb}$ ) prophages in the $B$. anthracis genome as target fragments. $B$. anthracis A16R genomic DNA was used as a template to amplify upstream and downstream regions of lambdaBa03 using primers Ulam03F/Ulam03R and Dlam03F/Dlam03R, respectively, for vector construction. The two fragments were inserted into the corresponding SalI and XbaI sites of pJOE899 (Altenbuchner, 2016). The resultant plasmid was then digested with $B s a \mathrm{I}$, and the large fragment (about $9 \mathrm{~kb}$ ) of digested plasmid was ligated with the small double-stranded DNA,annealed with the two complementary oligonucleotides sg-lam03F/sg-lam03R, to obtain plasmid pJOE-Lam03. Plasmid pJOE-Lam01, used for the deletion of prophage lambdaBa01, was constructed in the same way.

\footnotetext{
${ }^{1}$ https://sg.idtdna.com/site/order/designtool/index/CRISPR_CUSTOM
}

To construct a vector for introducing a point mutation into $p l c R$, a mutant $p l c R$ sequence containing a new termination codon, G640T, was synthesized by Genewiz (Suzhou, China). Five other same-sense mutation sites, C735G, A738T, G741C, G744A, and $\mathrm{G} 747 \mathrm{~A}$, were also introduced into the synthesized sequence. This fragment was then cloned into the two SfiI sites of plasmid pJOE8999. The resultant plasmid was digested by BsaI, and the large fragment of digested plasmid was ligated with the small double-stranded DNA, annealed with the two complementary oligonucleotides sg-plcRF/sg-plcRR, to obtain plasmid pJHRT.

\section{Construction and Isolation of B. anthracis Mutants}

The transformation and selection of $B$. anthracis A16R mutants was performed as described previously (Wang et al., 2018). The recombinant pJOE-based plasmids constructed above were introduced by electroporation into B. anthracis strain A16R. Transformants were selected at $30^{\circ} \mathrm{C}$ on BHIG medium containing kanamycin $(25 \mu \mathrm{g} / \mathrm{ml})$. Colonies were visible after $12-16 \mathrm{~h}$. A single colony was then inoculated into liquid medium supplemented with $25 \mu \mathrm{g} / \mathrm{ml}$ kanamycin and incubated with shaking for $3 \mathrm{~h}$ at $37^{\circ} \mathrm{C}$. Mannose (final concentration, $0.4 \%$, w/v) was then added to induce the expression of the Cas9 protein. After a further $3 \mathrm{~h}$ of cultivation, serial dilutions of the culture 
were plated on LB agar containing $25 \mu \mathrm{g} / \mathrm{ml}$ kanamycin and $0.4 \%$ mannose and incubated at $37^{\circ} \mathrm{C}$ overnight. Following incubation, at least nine colonies were validated by PCR using multi-pair primers. B. anthracis A16R was used as a control. To eliminate the plasmid for genome editing, mutant strains were passaged up to three times in the absence of antibiotics to ensure plasmid loss, which was confirmed by the loss of kanamycin resistance.

\section{Construction and Isolation of $B$. cereus Mutants}

Electrocompetent cells were prepared as described previously (Wang et al., 2018). Approximately $1 \mu \mathrm{g}$ of plasmid pJHRT, derived from E. coli $\mathrm{DH} 5 \alpha$, was mixed with electrocompetent B. cereus cells and pulsed $(0.6 \mathrm{kV}, 500 \Omega$, and $25 \mu \mathrm{F})$ in a $0.1 \mathrm{~cm}$ gap cuvette. The cells were immediately resuspended in $1 \mathrm{ml}$ of LB medium and incubated for $1 \mathrm{~h}$ at $30^{\circ} \mathrm{C}$ with shaking. Recovered cells were then spread on LB agar plates containing kanamycin $(25 \mu \mathrm{g} / \mathrm{ml})$.

Kanamycin-resistant $B$. cereus HN001 colonies were picked and assessed for the presence of recombinant plasmid pJHRT by PCR using the primer pair pJOEF/pJOER. Verified colonies were transferred to liquid medium supplemented with $25 \mu \mathrm{g} / \mathrm{ml}$ of kanamycin and incubated with shaking for $3 \mathrm{~h}$ at $30^{\circ} \mathrm{C}$. Mannose (final concentration, $0.4 \%$, w/v) was added to induce the expression of the Cas 9 protein. Following incubation for a further $13 \mathrm{~h}$ at $28^{\circ} \mathrm{C}$, the cultures were transferred to new liquid LB medium ( $1 \%$ dilution) and Cas9 expression was again induced. Finally, serial dilutions of the cultures were plated on sheep blood agar and incubated at $30^{\circ} \mathrm{C}$ overnight. Hemolysin activity was observed after $18 \mathrm{~h}$, and clones with very small zones of clearing (indicating hemolysis) were selected for PCR-based analysis using primers plcRHMF/plcRHMR, followed by sequencing. Plasmids for genome editing were eliminated as described above for $B$. anthracis. Mutants were further identified by analysis of hemolysin and phospholipase activities as described above.

\section{RESULTS}

\section{Excision of Two Prophages in B. anthracis}

The schematic shown in Figure $\mathbf{1}$ outlines prophage excision and the method used to integrate donor DNA, sgRNA, and cas9 cassettes into a single plasmid. The target site for Cas 9 was in the center of the lambdaBa03 prophage. Alternative mutants were detected by multiplex PCR using oligonucleotides lam03p1-lam03p5 (Figure 2). The region amplified by the lam03p1/lam03p2 primers in the control strain was $>19 \mathrm{~kb}$ in length, which exceeded the maximum amplification size under our PCR conditions. However, all randomly selected colonies (9/9) showed the expected $2.2 \mathrm{~kb}$ amplicon, indicating that the prophage locus had been deleted (Figure 2A). Genomic DNA was the extracted from four of the clones, and primers lam03p4/lam03p5 and lam03p1/lam03p3 were used for further PCR verification. No target sequences were amplified from the mutant strains, while amplicons were obtained from the control strain (Figures 2B,C). Because of the location of the primers (Figure 2D), the expected fragments (1.6 or $0.6 \mathrm{~kb}$, respectively) could only be amplified from the wild-type strain and not from the mutants. In addition, DNA sequencing of the fragments amplified by lam03p1/lam $03 \mathrm{p} 2$ primers confirmed that prophage lambdaBa03 was not present within that $2.2 \mathrm{~kb}$ amplicons (Figure 2E). Together, these results indicated that prophage lambdaBa03 had been completely eliminated in the mutant strains (Figures 2A-C,E).

Using a similar technique, we also excised the largest prophage, lambdaBa01, although with a relatively low success rate (2/9; Figures $3 \mathbf{A}-\mathbf{E})$. This efficiency was nevertheless higher than that achieved using other methods based on homologous recombination in B. anthracis.

\section{plcR Point Mutation in B. cereus}

Using $B$. cereus plcR as a target, we investigated the feasibility of this method to carry out point mutations. We designed the G640T mutation based on the sequence of non-functional B. anthracis plcR gene, resulting in a non-functional PlcR protein and loss of hemolytic activity. As described above, a plasmid containing mutant plcR sgRNA and cas9 cassettes was electroporated into $B$. cereus strain HN001. Using this method, we successfully obtained recombinant strains that did not produce an obvious zone of clearing on sheep blood agar, indicating loss of hemolytic activity (Figure 4A). PCR-based analysis and DNA sequencing of these strains confirmed that the plcR gene contained the G640T mutation (Figures 4B,C). Further hemolysin and phospholipase activity assays revealed partial loss of hemolysin activity and total loss of phospholipase activity in the three selected mutant isolates, named $B$. cereus HN1M. The characteristics of the mutant strain were similar to those of B. anthracis strain A16PI2 (Wang et al., 2011), which contains a naturally-occurring nonsense mutation in $p l c R$ (Figure 5).

\section{DISCUSSION}

Deletion of important virulence-related genes is a means of constructing live attenuated vaccines, however, the choice of mutation method is pivotal to this process (Racz et al., 2013; Kamble and Lee, 2016). Desirable mutants are free from screening marker residues and should have a plaque-free presence within the genome (Mignon et al., 2015). To date, this has been achieved by various anti-selective technologies (Reyrat et al., 1998). We previously used a modified pheS* counter-selection mutant construction system to obtain a mutant strain with no selective markers (Wang et al., 2018). However, subsequent experiments showed that the removal of resistance markers using $c l$-phe led to unexpected mutations, as well as the deletion of large genomic fragments near the designated mutation site (Wang et al., unpublished data). These phenomena revealed the limitations of this method and the need to optimize the protocol or develop alternative techniques.

Although the CRISPR/Cas9 system has been used successfully in Bacillus species, especially B. subtilis, there are physiological 
Large fragment deletion

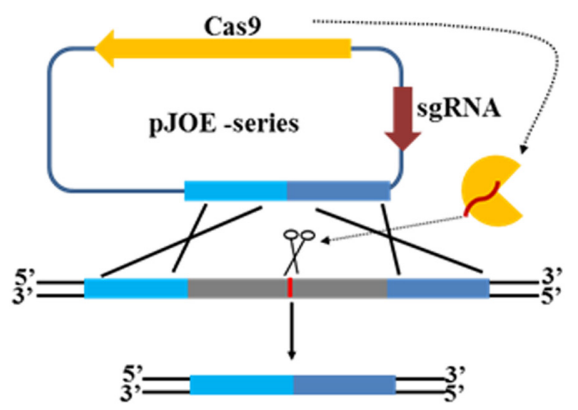

Site-specific mutagenesis

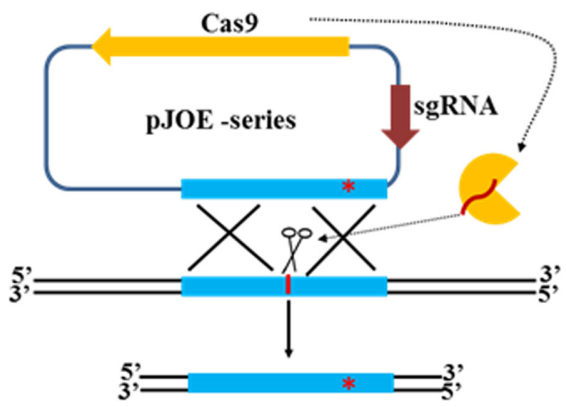

FIGURE 1 | Schematic representation of genome engineering in B. anthracis and B. cereus via the CRISPR/Cas9 system. The all-in-one CRISPR-Cas9 plasmids based on pJOE consist of Cas9, sgRNA, and upstream and downstream homologous arms that serve as donor DNA. Transcription of the sgRNA allows the Cas9 protein to cleave at a specific site in the genome. The desired mutation is then achieved by recombination between donor DNA and the genome.
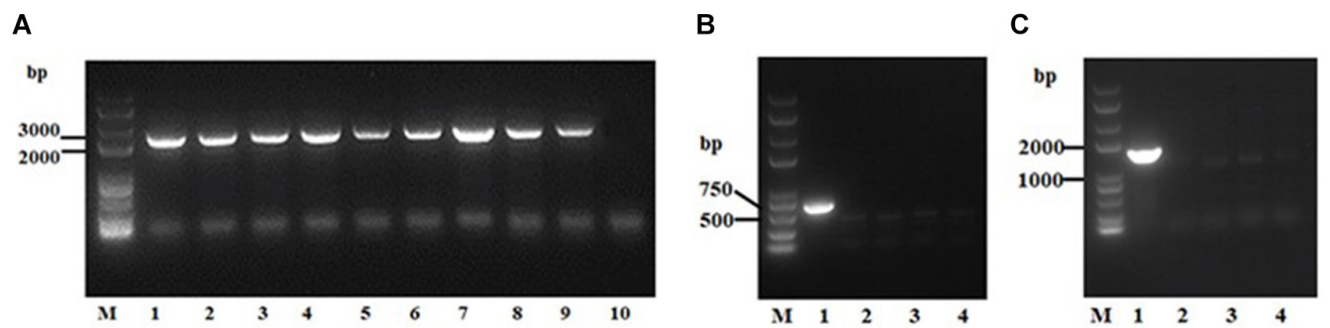

D

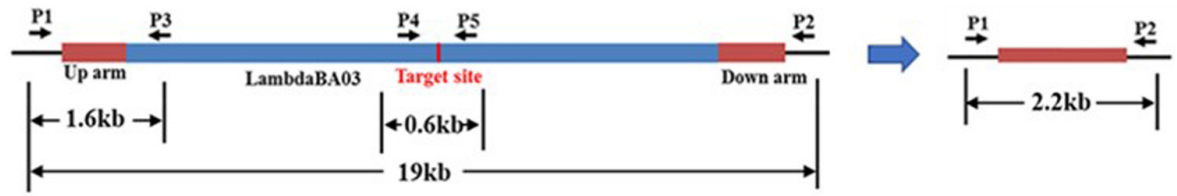

E

A16R: ACGGTCAGTA TTAC LambdaBa03(16.56kb) TTAT AATTATCGTT

Alam03: ACTTAgCAAT ACGgTCAgta aATTATCGTT TGATgtTATAA

TACT TAGCAATACG G T CAGTAA T TATC GT T T GAT TT TA A

FIGURE 2 | PCR-based verification of lambdaBa03 deletion in B. anthracis A16R. (A) Fragments amplified using primer pair lam03p1/lam03p2. The expected fragment in the mutant strain was approximately $2.2 \mathrm{~kb}$ (lanes $2-9$ ). The fragment in the wild-type strain was $>19 \mathrm{~kb}$, which exceeds the maximum amplification size under the PCR conditions used in this study (lane 1). (B,C) Fragments amplified using primer sets lam03p1/lam03p3 and lam03p4/lam03p5, respectively. Because of the location of the primers (D), the expected fragments (1.6 or $0.6 \mathrm{~kb}$, respectively) could only be amplified from the wild-type strain (lane 1) when the last two primer pairs were used for PCR (lane 1, A16R; lanes 2-4, mutant strains). (E) Sequencing data comparing wild-type strain A16R with the lambdaBa03 deletion mutant. 

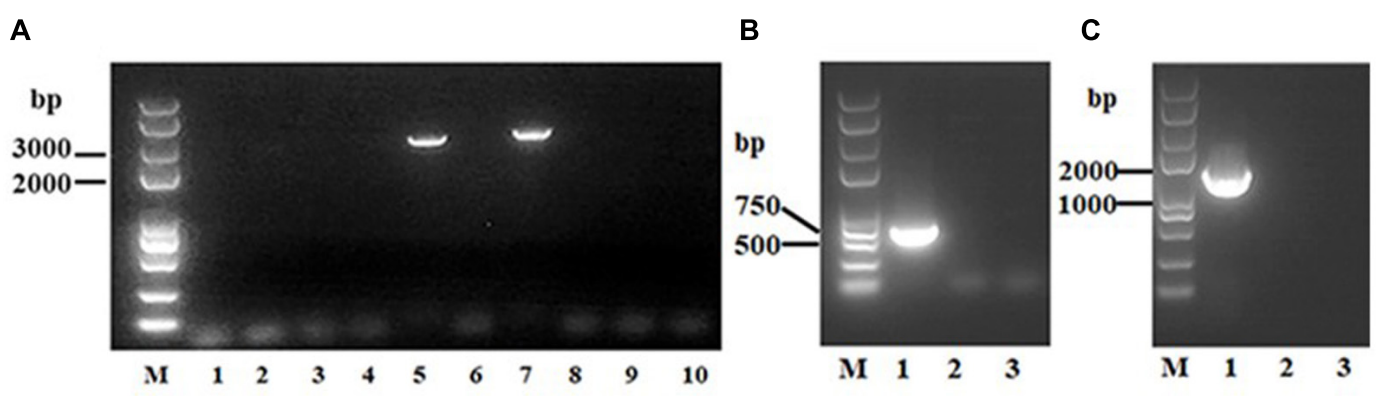

D

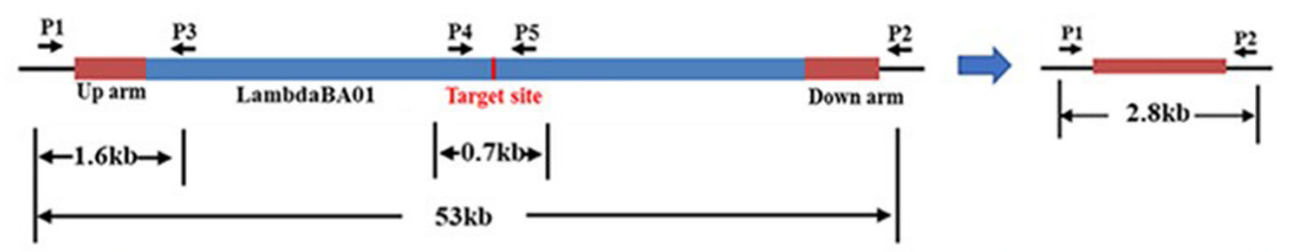

E
A16R: GGCTTCCTCA TCTG LambdaBa01(50.42kb)|TGAG CCTCTTAGT

Alam01:CCGTAGTATCAA GGCTTCCTCA CCTCTTAGTA AAGAGACATATA

C CGTAGTATCAAGGCTTCCTCACCTCTTAGTAAAGAGACATATA

Deletion site $\uparrow$

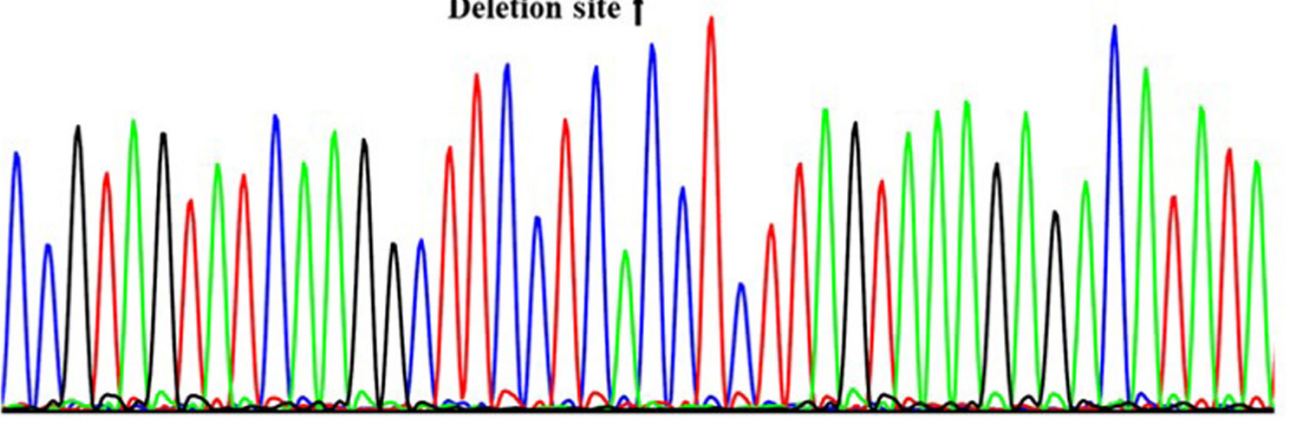

FIGURE 3 | PCR verification of lambdaBa01 deletion in B. anthracis A16R. (A) Fragments amplified using primer pair lam01p1/lam01p2. The expected fragment in the mutant strain was approximately $2.8 \mathrm{~kb}$ (lanes 5, 7). (B) Fragments amplified using primer pairs lam01p1/lam01p3 and lam01p4/lam01p5. (C) Because of the location of the primers (D), the expected fragments (1.6 or $0.7 \mathrm{~kb}$, respectively) could only be amplified from the wild-type strain (lane 1) and not from the mutants (lanes 2 and 3). (E) Sequencing data comparing wild-type strain A16R with the lambdaBa01 deletion mutant.

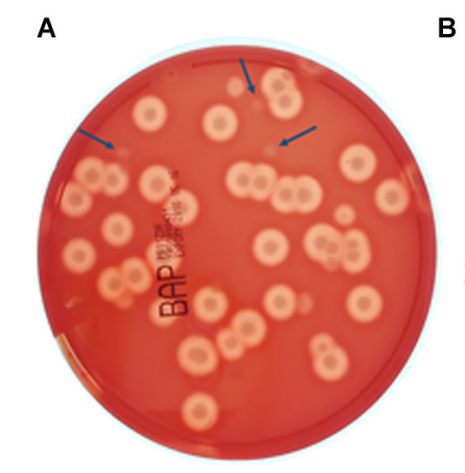

B

C

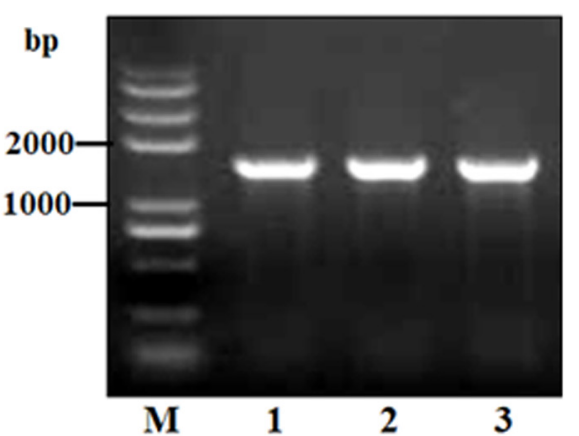

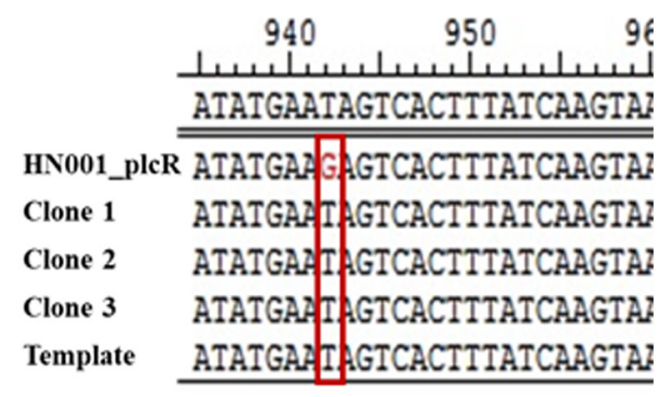

FIGURE 4 | Verification of site-specific mutagenesis of plcR in B. cereus HN001. (A) Selection of positive mutants using hemolysin assays. Clones indicated by blue arrows are candidates with poor hemolytic activity. (B) PCR-amplified fragment of plcR for sequencing. (C) Sequence analysis of plcR from the putative mutant clones and the wild-type strain HN001. All three candidate clones contained the G640T point mutation. 


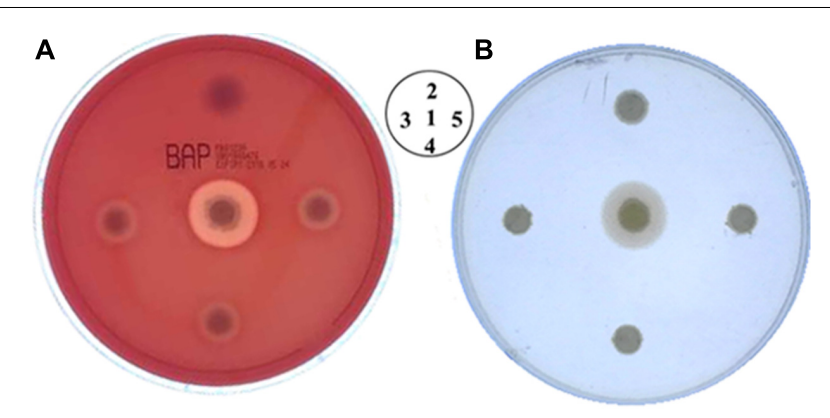

FIGURE 5 | Phenotypic analysis of B. cereus HN1M. (A) Hemolytic activity assay. (B) Phospholipase activity assay. Similar to $B$. anthracis, the hemolytic enzyme activity of positive mutants was partially lost, while phospholipase activity was completely lost. Different strain locations are shown. 1. B. cereus HN001, 2. B. anthracis A16PI2, 3-5. B. cereus HN1M.

differences between B. anthracis and B. subtilis. As such, many successful methods used in $B$. subtilis have not been implemented in B. anthracis. For example, single-stranded DNA oligonucleotide-mediated mutagenesis and PCR-based homologous recombination have been reported in many B. subtilis studies (Yan et al., 2008; Wang et al., 2012), but there are no corresponding reports in B. anthracis. Furthermore, our experiment also showed that the detail of execution process was not exactly the same. For example, the induction condition of Cas9 expression in B. anthracis and B. cereus was different. Therefore, it is imperative that we explore the feasibility of alternative systems in $B$. anthracis and B. cereus.

Our results confirm that the CRISPR/Cas9 system can be used to perform large-fragment deletions and precise point mutations in the genomes of $B$. anthracis and B. cereus, respectively. This work lays important foundations for the application of the method in related strains. Based on the current data and other published literature, there does not appear to be any significant difference in the success rate of the pJOE8999-based genome editing method between B. subtilis and B. anthracis. When the method was used to delete a large fragment $(25 \mathrm{~kb})$ in B. subtilis, $>90 \%$ of the tested colonies showed the expected deletion (Altenbuchner, 2016). In the current study, largefragment $(\sim 17 \mathrm{~kb})$ deletion mutants were achieved with a rate of $100 \%$. Similarly, $100 \%(72 / 72)$ of tested colonies showed the correct sequence when this system was used to repair a $3 \mathrm{bp}$ mutation of trpC2 in B. subtilis in the same article. However, when the same system was used for site-specific mutagenesis of plcR gene in B. cereus, less than 10\% (3/40) of clones contained the mutation, a mutation rate far lower than that regularly achieved in B. subtilis. Based on this finding, we thought the reason of this result is that $\mathrm{PlcR}$ is a fundamental regulatory protein in B. anthracis and likely plays an almost indispensable role in bacterial survival. To improve the efficiency of gene editing in $B$. cereus group strains, further optimization of the design of the gRNA, the mannose concentration, and the length of the homologous arms should be conducted.

The major shortcoming of the CRISPR/Cas9 system is the potential for off-target modification caused by repair of double-stranded chromosomal breaks through non-homologous end joining (NHEJ). Although a NHEJ system has been identified in B. subtilis (Weller et al., 2002; Moeller et al., 2011), published data indicate that the NHEJ system in B. subtilis is only active during sporulation, and therefore not active in growing cells (Moeller et al., 2011; Zheng et al., 2017). In addition, Toymentseva and Altenbuchner (2019) confirmed that the NHEJ system was not activated when CRISPR/Cas9-mediated genome editing was carried out in B. subtilis. No equivalent NHEJ system has been identified experimentally the $B$. cereus group strains investigated in the current study, although NHEJ-associated proteins (such as Ku-like proteins) have been identified by homology analysis. According to published research, NHEJmediated repair mechanisms in $B$. anthracis are inefficient compared with those found in B. subtilis (Cote et al., 2018). Therefore, strains with non-specific cleavage might not survive in the absence of homologous arms to repair the genomic DNA breaks. This characteristic would ensure that almost all of the resulting clone contained the correct modification. Nevertheless, future studies should carry out genome-wide sequencing of the strains to analyze possible off-target effects and determine the reliability of the system.

Using $B$. anthracis, we eliminated two different prophages using CRISPR/Cas9 technology, confirming that this system is a meaningful genetic manipulation tool for research in this important human pathogen. Although deletion of large fragments of the $B$. anthracis genome has been reported, the protocol used was very complex (Pomerantsev et al., 2006). To accomplish deletion, genes on either side of the target fragment were deleted by means of homologous recombination and the Cre/LoxP system, and loxP sites were introduced at both ends of the target fragment. Recombination at the loxP sites by Cre recombinase resulted in deletion of the large intervening region. However, the loxP sites remain in the genome, which may affect the long-term stability of the bacterial genome. Although such genetic instability can be minimized using mutational lox sites (lox66 and lox71), scars (lox72) still remain in the target genome (Lambert et al., 2007). Overall, our method is far simpler than the Cre/LoxP method, and the correspondingly shorter experimental period is valuable for mutant construction.

We also successfully introduced a G640T point mutation into $p l c R$ in $B$. cereus. This technique could be used to study the specific regulatory mechanisms of important virulence-related genes in B. cereus. In most Bacillus strains, the products of $p l c R$ and its related genes have important physiological functions. Yet in $B$. anthracis, the most virulent of the $B$. cereus group species, PlcR is non-functional (Mignot et al., 2001; Gohar et al., 2008; Bhunia, 2018). The mutant strain developed in the current study could therefore be used as a possible model strain to explore why B. anthracis containing a nonsense mutation at position 640 forms a stop codon that fails to produce a functional $\mathrm{PlcR}$ protein.

Therefore, we have developed a simple and fast genetic manipulation tool for the study of $B$. anthracis. As well as being a method for label-free deletion of large fragments in $B$. anthracis, providing a new and effective technique for constructing live attenuated vaccine strains, this technique also allows us to obtain recombinant strains with precise deletions of the prophages. 
This could provide the necessary model strains for functional study of genes carried by these prophages in the growth, reproduction, and pathogenic process of $B$. anthracis. These studies would ultimately help to reveal the biological importance of the B. anthracis-specific prophages.

\section{DATA AVAILABILITY}

The raw data supporting the conclusions of this manuscript will be made available by the authors, without undue reservation, to any qualified researcher.

\section{AUTHOR CONTRIBUTIONS}

YW, XL, and CL designed the research. YW, DW, XW, HT, EF, LZ, CP, and BW performed all experiments. CL and HW

\section{REFERENCES}

Acevedo, M. M., Carroll, L. M., Mukherjee, M., Mills, E., Xiaoli, L., Dudley, E. G., et al. (2019). Bacillus clarus sp. nov. is a new Bacillus cereus group species isolated from soil. BioRxiv

Altenbuchner, J. (2016). Editing of the Bacillus subtilis genome by the CRISPR-Cas9 system. Appl. Environ. Microbiol. 82, 5421-5427. doi: 10.1128/AEM.01453-16

Argôlo-Filho, R., and Loguercio, L. (2014). Bacillus thuringiensis is an environmental pathogen and host-specificity has developed as an adaptation to human-generated ecological niches. Insects 5, 62-91. doi: 10.3390/insects 5010062

Bhunia, A. K. ed. (2018). Bacillus cereus and Bacillus anthracis. in Foodborne Microbial Pathogens. (New York, NY: Springer).

Chandrasekaran, A. P., Song, M., and Ramakrishna, S. (2017). Genome editing: a robust technology for human stem cells. Cell. Mol. Life Sci. 74, 3335-3346. doi: 10.1007/s00018-017-2522-0

Cong, L., Ran, F. A., Cox, D., Lin, S., Barretto, R., Habib, N., et al. (2013). Multiplex genome engineering using CRISPR/Cas systems. Science 339, 819-823. doi: 10.1126/science. 1231143

Cote, C. K., Buhr, T., Bernhards, C. B., Bohmke, M. D., Calm, A. M., EstebanTrexler, J. S., et al. (2018). A standard method to inactivate Bacillus anthracis spores to sterility via gamma irradiation. Appl. Environ. Microbiol. 84, e106e118. doi: 10.1128/AEM.00106-18

Dong, H., and Zhang, D. (2014). Current development in genetic engineering strategies of Bacillus species. Microb. Cell.Fact. 13, 63. doi: 10.1186/1475-285913-63

Gersbach, C. A., Gaj, T., and Barbas, C. F. (2014). Synthetic zinc finger proteins: the advent of targeted gene regulation and genome modification technologies. Accounts Chem. Res. 47, 2309-2318. doi: 10.1021/ar500039w

Gohar, M., Faegri, K., Perchat, S., Ravnum, S., Økstad, O. A., Gominet, M., et al. (2008). The PlcR virulence regulon of Bacillus cereus. PLoS One. 3:e2793. doi: 10.1371/journal.pone.0002793

Granum, P. E. (2017). Spotlight on Bacillus cereus and its food poisoning toxins. FEMS Microbiol. Lett. 364, fnx071.

Guinebretière, M. H., Thompson, F. L., Sorokin, A., Normand, P., Dawyndt, P., Ehling-Schulz, M., et al. (2008). Ecological diversification in the Bacillus cereus group. Environ. Microbiol. 10, 851-865. doi: 10.1111/j.1462-2920.2007. 01495.x

Guo, Z., Sun, D., Kang, S., Zhou, J., Gong, L., Qin, J., et al. (2019). CRISPR/Cas9mediated knockout of both the PxABCC2 and PxABCC3 genes confers highlevel resistance to Bacillus thuringiensis CrylAc toxin in the diamondback moth, plutella xylostella (L.). Insect Biochem. Mol. Biol. 107, 31-38. doi: 10.1016/ j.ibmb.2019.01.009

Joung, J. K., and Sander, J. D. (2013). TALENs: a widely applicable technology for targeted genome editing. Nat. Rev. Mol. Cell Bio. 14, 49. doi: 10.1038/nrm3486 analyzed data. YW, XL, and HW wrote the manuscript. All authors reviewed the final manuscript.

\section{FUNDING}

This work was supported by the State Major Science and Technology Special Projects of China (Grant No. 2018ZX10714002) and the National Natural Science Foundation of China (Grant Nos. 81571958, 81671979, and 81601739).

\section{ACKNOWLEDGMENTS}

We thank Sarah Williams, Ph.D., and Tamsin Sheen, Ph.D., from Liwen Bianji, Edanz Group China (www.liwenbianji.cn), for editing the English text of a draft of this manuscript.

Kamble, N. M., and Lee, J. H. (2016). Characterization and Evaluation of a Salmonella enterica Serotype senftenberg mutant created by deletion of virulence-related genes for use as a live attenuated vaccine. Clin. Vaccine Immunol. 23, 802-812. doi: 10.1128/cvi.00233-16

Kotiranta, A., Lounatmaa, K., and Haapasalo, M. (2000). Epidemiology and pathogenesis of Bacillus cereus infections. Microbes Infect. 2, 189-198. doi: 10.1016/s1286-4579(00)00269-0

Lambert, J. M., Bongers, R. S., and Kleerebezem, M. (2007). Cre-lox-based system for multiple gene deletions and selectable-marker removal in Lactobacillus plantarum. Appl. Environ. Microbiol. 73, 1126-1135. doi: 10.1128/aem.01473-06

Li, K., Cai, D., Wang, Z., He, Z., and Chen, S. (2018). Development of an efficient genome editing tool in Bacillus licheniformis using CRISPR-Cas9 nickase. Appl. Environ. Microbiol. 84, e2608-e2617. doi: 10.1128/AEM.026 08-17

Liu, Y., Lai, Q., Göker, M., Meier-Kolthoff, J. P., Wang, M., Sun, Y., et al. (2015). Genomic insights into the taxonomic status of the Bacillus cereus group. Sci. Rep. 5, 14082. doi: 10.1038/srep14082

Maeder, M. L., and Gersbach, C. A. (2016). Genome-editing technologies for gene and cell therapy. Mol. Ther. 24, 430-446. doi: 10.1038/mt.2016.10

Mignon, C., Sodoyer, R., and Werle, B. (2015). Antibiotic-free selection in biotherapeutics: now and forever. Pathogens 4, 157-181. doi: 10.1038/mt.2016.10

Mignot, T., Mock, M., Robichon, D., Landier, A., Lereclus, D., and Fouet, A. (2001). The incompatibility between the PlcR-and AtxA-controlled regulons may have selected a nonsense mutation in Bacillus anthracis. Mol. Microbiol. 42, 1189-1198. doi: 10.1046/j.1365-2958.2001.02692.x

Moeller, R., Schuerger, A. C., Reitz, G., and Nicholson, W. L. (2011). Impact of two DNA repair pathways, homologous recombination and non-homologous end joining, on bacterial spore inactivation under simulated martian environmental conditions. Icarus 215, 204-210. doi: 10.1016/j.icarus.2011.06.035

Okinaka, R. T., and Keim, P. (2016). "The phylogeny of Bacillus cereus sensu lato," in The Bacterial Spore: from Molecules to Systems, eds A. Driks, and P., Eichenberger (Washington, D.C.: American Society of Microbiology), 239-251.

Pilo, P., and Frey, J. (2018). Pathogenicity, population genetics and dissemination of Bacillus anthracis. Infect. Genet. Evol. 64, 115-125. doi: 10.1016/j.meegid. 2018.06.024

Plaut, R. D., and Stibitz, S. (2015). Improvements to a markerless allelic exchange system for Bacillus anthracis. PLoS One 10:e0142758. doi: 10.1371/journal.pone. 0142758

Pomerantsev, A. P., Sitaraman, R., Galloway, C. R., Kivovich, V., and Leppla, S. H. (2006). Genome engineering in Bacillus anthracis using Cre recombinase. Infect. Immun. 74, 682-693. doi: 10.1128/iai.74.1.682-693.2006

Racz, R., Chung, M., Xiang, Z., and He, Y. (2013). Systematic annotation and analysis of "virmugens"-Virulence factors whose mutants can be used as live attenuated vaccines. Vaccine 31, 797-805. doi: 10.1016/j.vaccine.2012. 11.066 
Reyrat, J. M., Pelicic, V., Gicquel, B., and Rappuoli, R. (1998). Counterselectable markers: untapped tools for bacterial genetics and pathogenesis. Infect. Immun. 66, 4011-4017.

Rossi, G. A. M., Silva, H. O., Aguilar, C. E. G., Rochetti, A. L., Pascoe, B., Méric, G., et al. (2017). Comparative genomic survey of Bacillus cereus sensu stricto isolates from the dairy production chain in Brazil. FEMS Microbiol. Lett. 365, fnx283. doi: 10.1093/femsle/fnx283

Tan, T. T., Zhang, X. D., Miao, Z., Yu, Y., Du, S. L., Hou, X. Y., et al. (2019). A single point mutation in hmgA leads to melanin accumulation in Bacillus thuringiensis BMB181. Enzyme Microb. Tech. 120, 91-97. doi: 10.1016/j.enzmictec.2018. 10.007

Toymentseva, A. A., and Altenbuchner, J. (2019). New CRISPR-Cas9 vectors for genetic modifications of Bacillus species. FEMS Microbiol. Lett. 366, fny 284. doi: 10.1093/femsle/fny284

Wang, H. G., Liu, X. K., Feng, E. L., Zhu, L., Wang, D. S., Liao, X., et al. (2011). Curing the plasmid pXO2 from Bacillus anthracis A16 using plasmid incompatibility. Curr. Microbiol. 62, 703-709. doi: 10.1007/s00284-0109767-2

Wang, P., Zhu, Y., Zhang, Y., Zhang, C., Xu, J., Deng, Y., et al. (2016). Mob/oriT, a mobilizable site-specific recombination system for unmarked genetic manipulation in Bacillus thuringiensis and Bacillus cereus. Microb. Cell Fact. 15, 108. doi: 10.1186/s12934-016-0492-9

Wang, Y., Weng, J., Waseem, R., Yin, X. H., Zhang, R. F., and Shen, Q. R. (2012). Bacillus subtilis genome editing using ssDNA with short homology regions. Nucleic Acids Res. 40, e91. doi: 10.1093/nar/gks248

Wang, Y. C., Yuan, L. S., Tao, H. X., Jiang, W., and Liu, C. J. (2018). pheS* as a counter-selectable marker for marker-free genetic manipulations in Bacillus anthracis. J. Microbiol. Meth. 151, 35-38. doi: 10.1016/j.mimet.2018. 05.024
Weller, G. R., Kysela, B., Roy, R., Tonkin, L. M., Scanlan, E., Della, M., et al. (2002). Identification of a DNA nonhomologous end-joining complex in bacteria. Science 297, 1686-1689. doi: 10.1126/science.1074584

Westbrook, A. W., Moo-Young, M., and Chou, C. P. (2016). Development of a CRISPR-Cas9 tool kit for comprehensive engineering of Bacillus subtilis. Appl. Environ. Microbiol. 82, 4876-4895. doi: 10.1128/AEM.01159-16

Yan, X., Yu, H. J., Hong, Q., and Li, S. P. (2008). Cre/lox system and PCRbased genome engineering in Bacillus subtilis. Appl. Environ. Microbiol. 74, 5556-5562. doi: 10.1128/AEM.01156-08

Zhang, F., Wen, Y., and Guo, X. (2014). CRISPR/Cas9 for genome editing: progress, implications and challenges. Hum. Mol. Genet. 23, R40-R46. doi: 10.1093/hmg/ddu125

Zheng, X., Li, S. Y., Zhao, G. P., and Wang, J. (2017). An efficient system for deletion of large DNA fragments in Escherichia coli via introduction of both Cas9 and the non-homologous end joining system from Mycobacterium smegmatis. Biochem. Bioph. Res. Commun. 485, 768-774. doi: 10.1016/j.bbrc. 2017.02.129

Conflict of Interest Statement: The authors declare that the research was conducted in the absence of any commercial or financial relationships that could be construed as a potential conflict of interest.

Copyright (c) 2019 Wang, Wang, Wang, Tao, Feng, Zhu, Pan, Wang, Liu, Liu and Wang. This is an open-access article distributed under the terms of the Creative Commons Attribution License (CC BY). The use, distribution or reproduction in other forums is permitted, provided the original author(s) and the copyright owner(s) are credited and that the original publication in this journal is cited, in accordance with accepted academic practice. No use, distribution or reproduction is permitted which does not comply with these terms. 